\title{
Impact of Turbulence Models on the Air Flow in a Confined Rectangular Space
}

\author{
Jakub Mularski ${ }^{*} \mathbb{D}^{\mathbb{D}}$, Amit Arora $^{2}$, Muhammad Azam Saeed $^{3} \mathbb{D}^{\mathbb{D}}$, Lukasz Niedzwiecki $^{1}$ (D) \\ ${ }^{1}$ Wroclaw University of Science and Technology, Faculty of Mechanical and Power Engineering, 27 Wybrzeze Wyspianskiego St, 50- \\ 370 Wroclaw, Poland \\ ${ }^{2}$ Shaheed Bhagat Singh State Technical Campus, Ferozepur-152004, Punjab, India. \\ ${ }^{3}$ Department of Chemical Engineering, University of Engineering and Technology, Faisalabad Campus, Lahore, Pakistan \\ Email: jakub.mularski@pwr.edu.pl
}

Received: 4 September 2020; Revised: 12 October 2020; Accepted: 13 October 2020

\begin{abstract}
The paper regards the impact of four different turbulence models on the air flow pattern in a confined rectangular space. The following approaches are analyzed. The Baseline (BSL) Reynolds model, the Speziale-SarkarGatzki (SSG) Reynolds model, the Menter's shear-stress transport (SST) model and the basic $k-\varepsilon$ model. Computational fluid dynamics (CFD) results are compared with the experimental measurements in four different planes. The Reynolds number for the given conditions is equal to 5000. The $k-\varepsilon$ model yielded the most accurate results with regard to the experimental data but its reliability decreased near the wall region. With respect to the other models, it was also found that the $k-\varepsilon$ approach generated the least circulating flow.
\end{abstract}

Keywords: CFD, turbulence, air flow

\section{Introduction}

Computational fluid dynamic (CFD) modelling enables to obtain comprehensive data regarding the specific process and allows one to understand the governing phenomena behind it. It also eliminates the need for complex experimental measurements which can be expensive and time consuming. It is a powerful tool that can model multiphase, turbulent and reacting flows. However, in order to obtain credible information regarding the analyzed case, such a complex simulation would require to incorporate specific sub-models which accurately describe the process in terms of thermodynamics, fluid mechanics, chemistry and physics.

The present paper is focused mainly on the issue of fluid mechanics. It considers a turbulent air flow in a confined space [1-11]. The topic is important and can be referred mostly to the air flow within buildings. The aim of the study is to assess the impact of examined turbulent models on the air flow pattern with regard to the experimental data and to understand which models yield the most reasonable results. An accurate description of the turbulent flow is, in fact, a fundamental step in computational fluid dynamic modelling. Based on the literature, no specific model was found that managed to be the most accurate in each analyzed process. Every model has its own specific features that makes it more or less appropriate for the specific case.

Many turbulence modelling approaches have been developed so far. The most widely used ones are based on the Reynolds averaged Navier-Stokes (RANS) equations: $k-\varepsilon$, BSL Reynolds, SST, SSG Reynolds. The $k-\varepsilon$ model [3] is

Copyright (C2020 Jakub Mularski, et al.

DOI: https://doi.org/10.37256/est.212021627

This is an open-access article distributed under a CC BY license

(Creative Commons Attribution 4.0 International License)

https://creativecommons.org/licenses/by/4.0/ 
one of the most common models. It is a two equation approach which includes two extra turbulent properties of the flow: turbulent kinetic energy- $k$ and eddy dissipation rate- $\varepsilon$. They both determine the energy and scale of turbulence. The $k-\varepsilon$ model has been shown to be accurate for free-shear layer flows with small pressure gradients and for wallbounder internal flows with small pressure gradients [12]. The SST approach [13] is also a two equation eddyviscosity turbulence model that combines $k-\omega$ model and $k-\varepsilon$ model. $K-\omega$ is used in the inner region of the boundary layer, whereas $k-\varepsilon$ is applied in the free shear flow. BSL Reynolds [14] model and SSG Reynolds [15] model solve the Reynolds stress transport equations. It allows to model the turbulence transport in a more accurate way. The BSLRSM is an $\omega$ based RSM which models the transport of turbulent dispersion in the same way as SST model (by adopting a blending function). The pressure-strain correlation is an important factor in the RSM approach. It describes the distribution of energy among the Reynolds stresses, the fluctuating velocity field and the mean velocity field. The SSG Reynolds incorporates a more advanced approach for modelling of the pressure-strain correlation. It is meant to describe more accurately the redistribution mechanism of the Reynolds stresses that leads the turbulence to isotropy [15]. These RANS-based turbulence models are relatively robust and numerically stable approaches which also reduce the computational effort of the simulation in comparison with such approaches as large eddy simulation (LES) or direct numerical simulation (DNS). The present paper analyzes the RANS method with the aforementioned $k-\varepsilon$, BSL Reynolds, SST and SSG Reynolds turbulence models. The structure of the paper is as follows: Section 2 considers the model setup. Section 3 handles the results and discussion. Section 4 handles an additional quantitative assessment of the accuracy of a particular turbulence model through the error analysis of the Figures 3-6 with regard to the experimental data. Section 5 considers conclusions.

\section{Model set-up}

This section describes the geometry and boundary conditions. The analysis is performed on a $2 \mathrm{D}$ domain by using a CFD software Ansys CFX. Gas phase is modelled assuming an Eulerian approach. The flow is assumed to be incompressible. Both the continuity equation and RANS equation are solved. The SIMPLE algorithm is used for pressure-velocity coupling. Second-order schemes are used for spatial discretization. The geometry is presented in Figure 1.

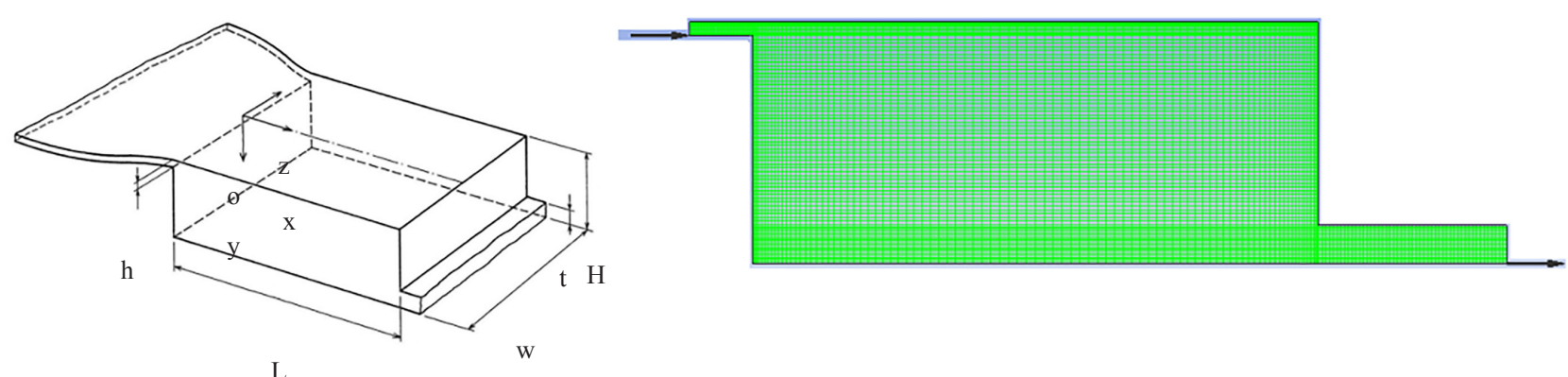

Figure 1. 2D Simulating mesh with the geometry [1]

The geometry has the following specification:

$$
L=9 \mathrm{~m}, H=3 \mathrm{~m}, h=0.168 \mathrm{~m}, t=0.48 \mathrm{~m}, w=1(2 \mathrm{D} \text { case })
$$

Inlet conditions are given by the velocity and the Reynolds number:

$$
u_{0}=0.455 \frac{m}{s}, \operatorname{Re}=5000
$$

where the kinematic viscosity $v=15.3 \times 10^{-6} \mathrm{~m}^{2} / \mathrm{s}$ at the inlet temperature of $20^{\circ} \mathrm{C}$. 
The mesh was generated in ICEM software. It is a structured, rectangular and non-uniform mesh consisting of 20 770 nodes. The mesh has been additionally refined near the walls.

In the case of boundary conditions a symmetry-type condition was set for the biggest side surface. Inlet was assigned with the velocity inlet condition with the normal velocity equal to $0,445 \mathrm{~m} / \mathrm{s}$. Outlet was assigned with the Outlet type condition with the average static pressure equal to $0 \mathrm{~Pa}$. For rest of the surfaces a wall-type condition was set. Walls were considered to be with no slip and smooth.

The experimental measurements, which the numerical analysis is based on, are made by laser-doppler anemometry. They were performed by [16].

\section{Results and discussion}

In order to obtain convergence, the following number of iterations were required:

$k-\varepsilon:$ 689, SST $k-\omega: 1106$, Baseline (BSL) Reynolds stress: 2147, Speziale-Sarkar-Gatski (SSG): 1767.

As for monitoring the convergence, residuals were set to $10^{-6}$ for all equations.

Figure 2 depicts velocity streamlines for each turbulent model. One may observe a similarity between the $k-\varepsilon$ model and the shear-stress transport (SST) approach. On the other hand, entirely different velocity field is predicted by the BSL-RSM and SSG-RSM. Additionally, BSL-RSM, SSG-RSM and the SST approach were found to generate much more intense circulating flow than the $k-\varepsilon$ approach. To further determine which field correctly corresponds to reality, Figures 3-6 present the relative velocity field in 4 planes predicted by the analyzed turbulence models with respect to the experimental data. The $k-\varepsilon$ model, despite its simplicity, manages to reproduce the most accurate results in each plane with regard to the rest of the models. In Figure 3, none of the adopted models were able to generate a velocity profile ideally similar to one obtained from experimental measurements. The $k-e$ approach, even though it reproduced the results with relatively highest accuracy, failed to predict the results appropriately near the wall region. In fact, each of the models overpredicted the velocity value close to the walls. In Figure 4, the velocity profiles obtained by the $k-\varepsilon$ model and the SST $k-\omega$ model were in relatively close agreement with the experimental data. The BSL approach significantly overpredicted the velocity close to the wall, while the SSG-RSM underpredicted its value. In the case of Figure 5, the best compliance with experiment was achieved by the $k-\varepsilon$ approach. The BSL model reproduced the results with the worst accuracy. Judging by Figure 6, the best agreement with experimental measurements was obtained by the SST model and the $k-\varepsilon$ model. The BSL approach showed the worst accuracy.
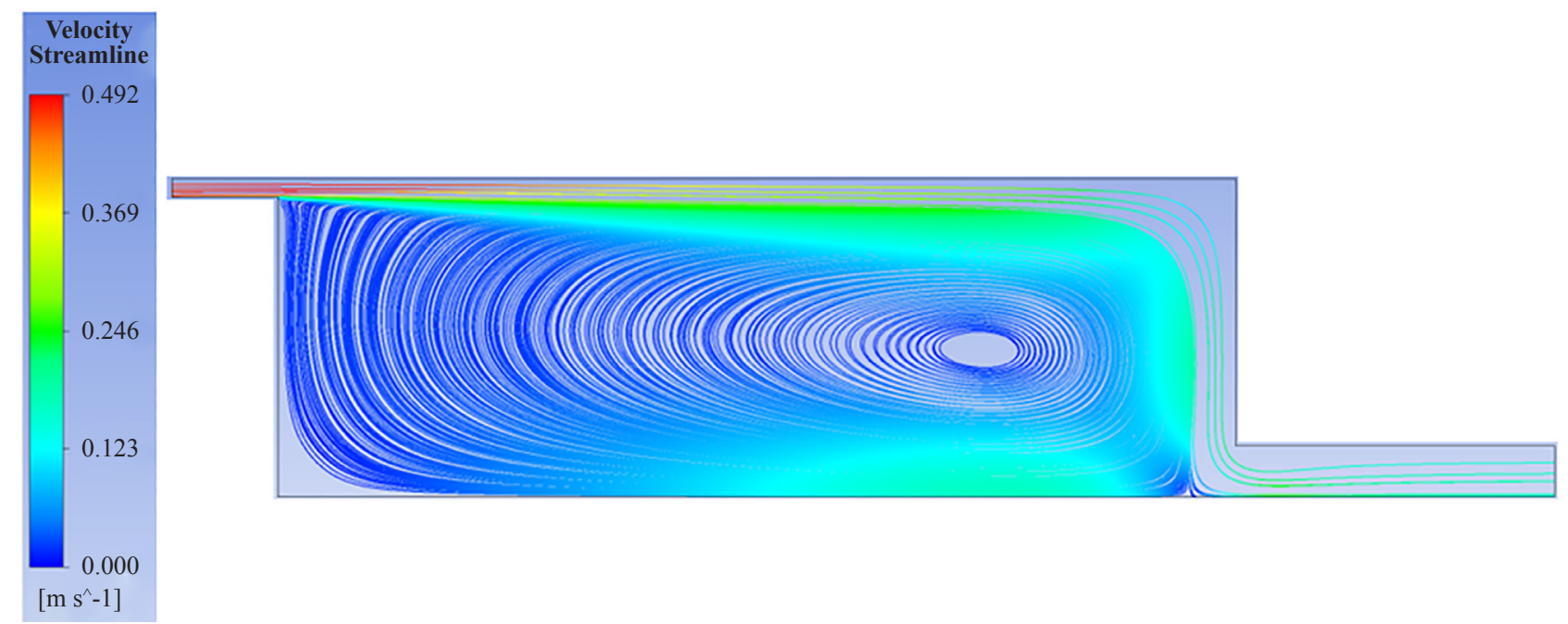
SST model

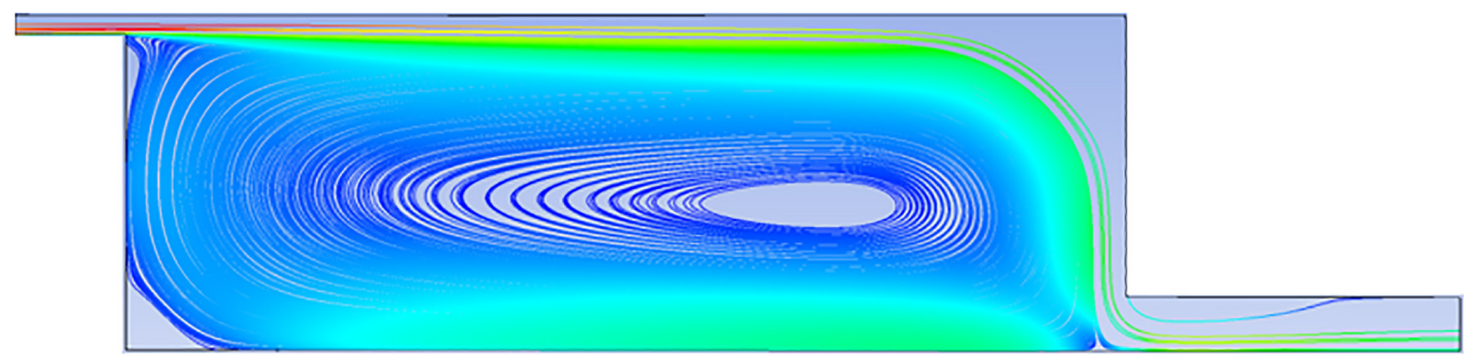

BSL Reynolds model

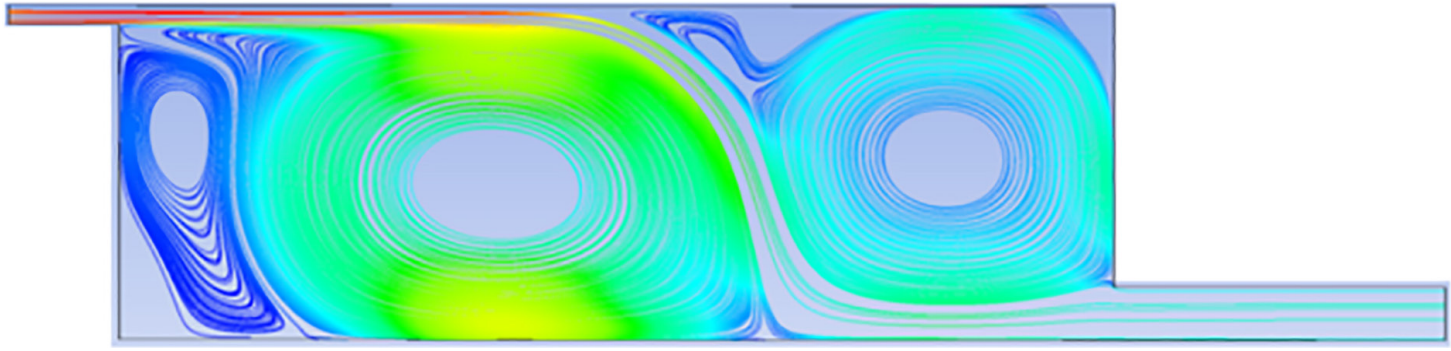

SSG Reynolds model

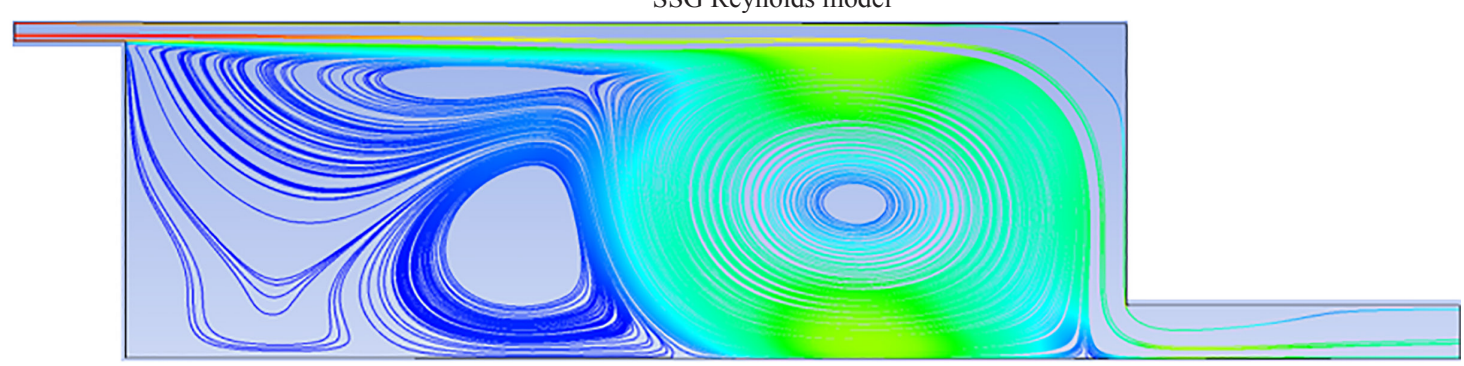

Figure 2. Velocity streamlines for each turbulent model

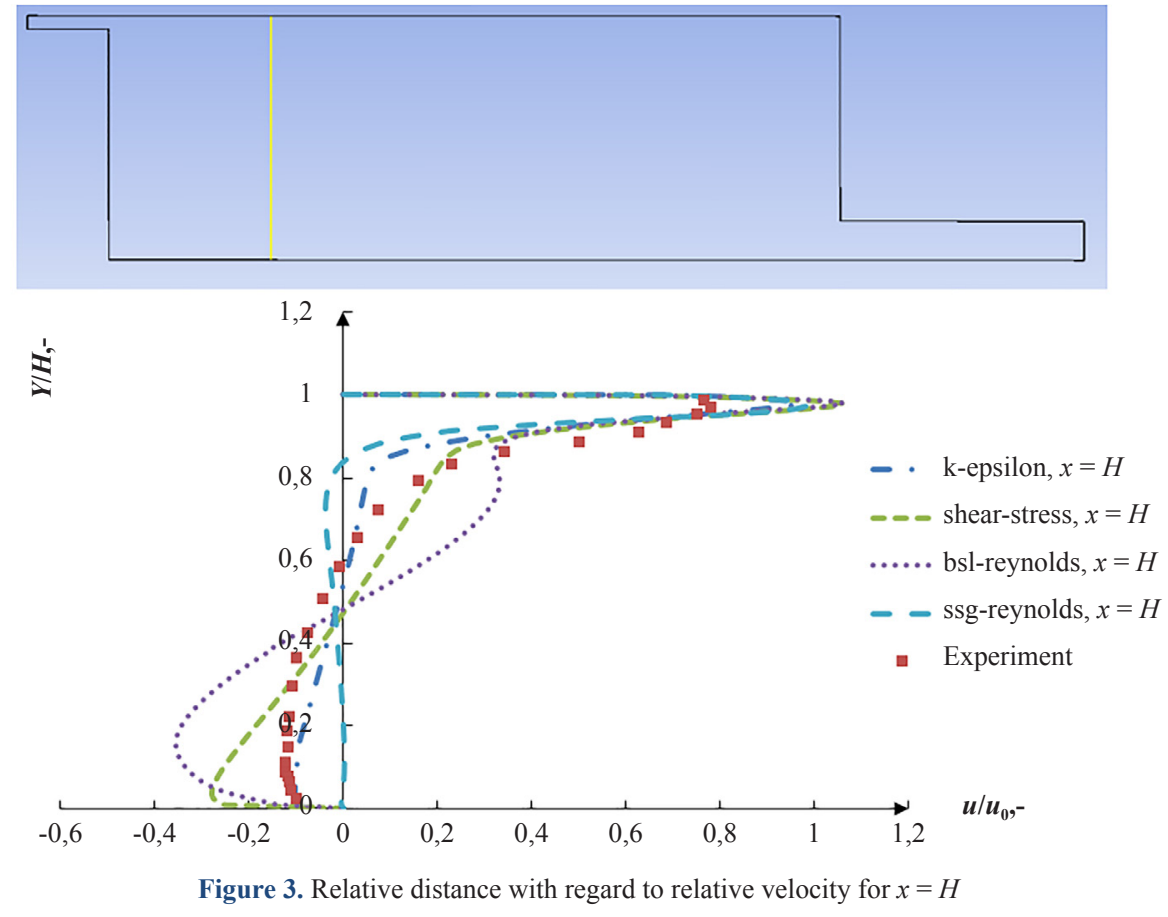



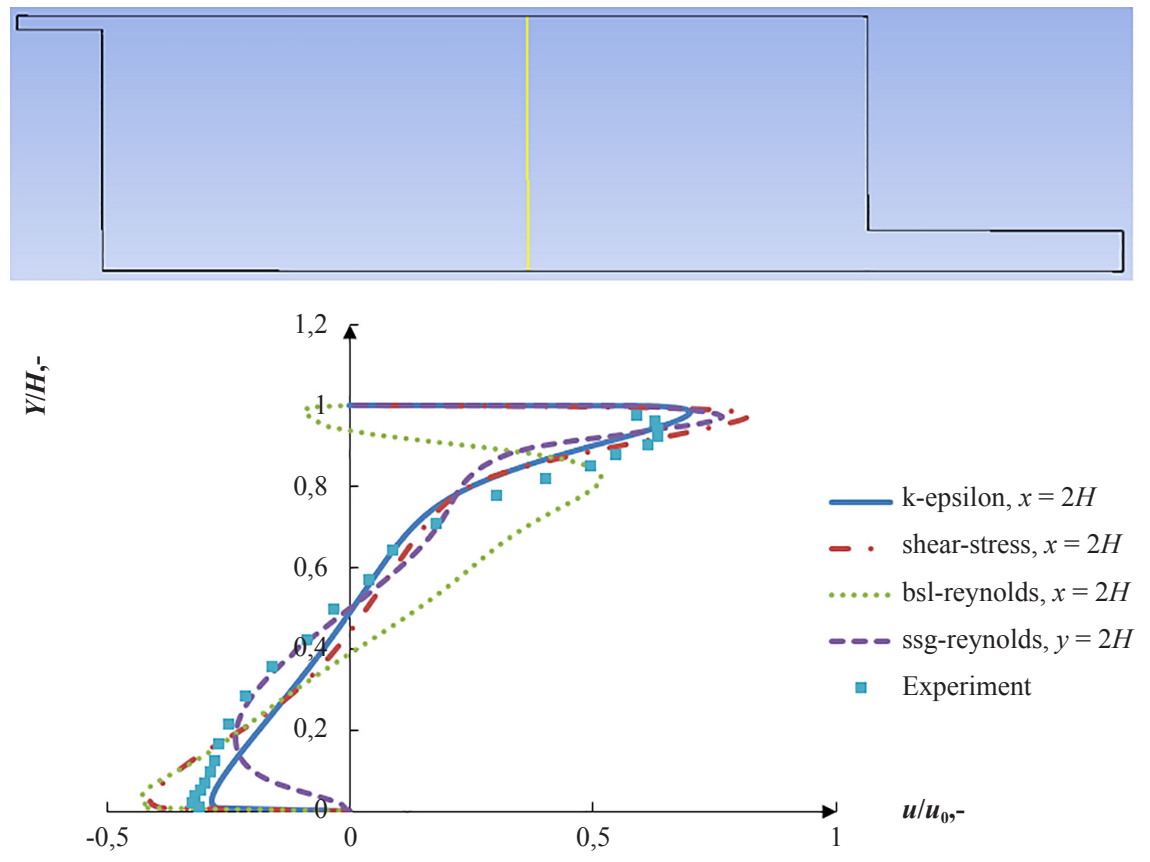

Figure 4. Relative distance with regard to relative velocity for $x=2 H$
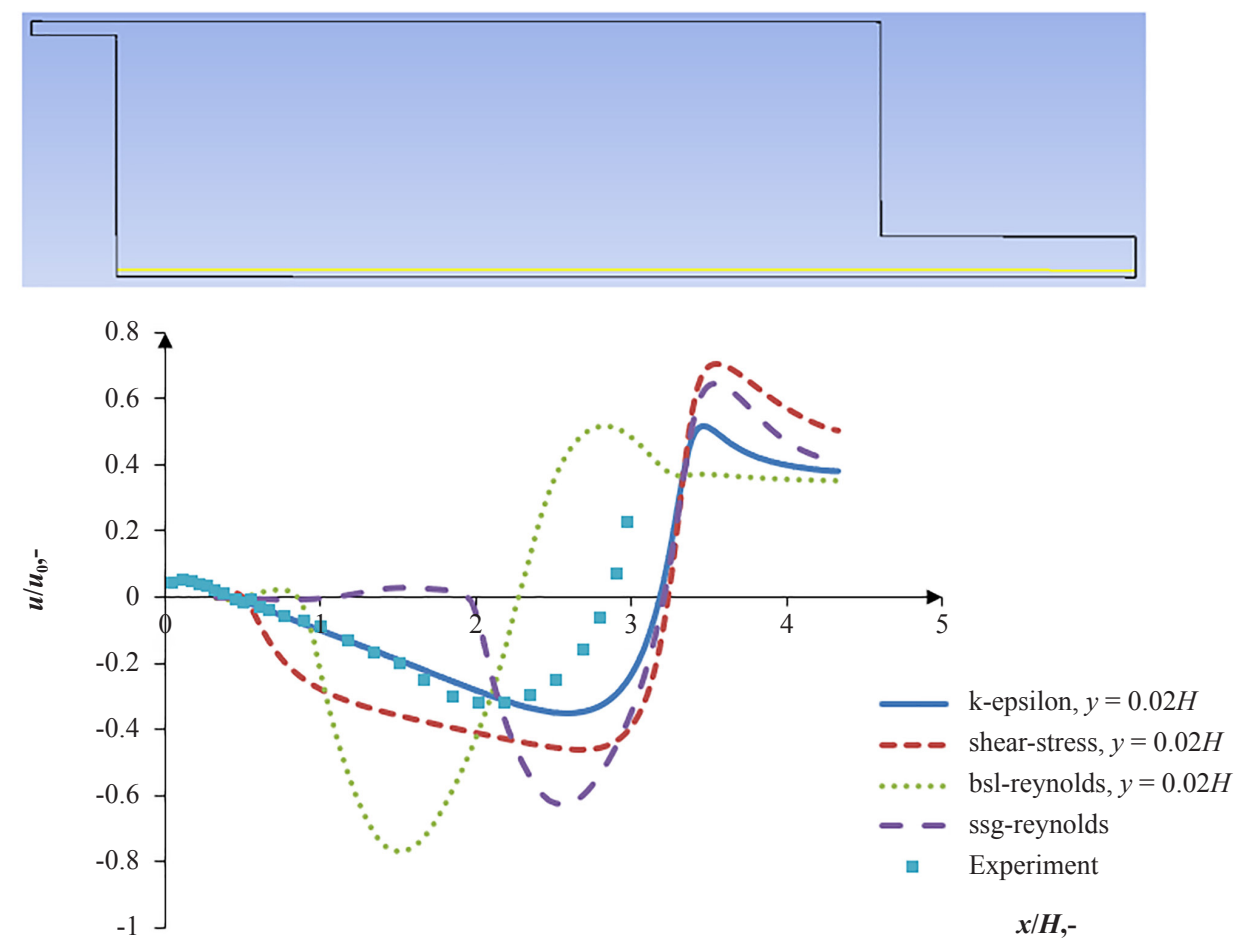

Figure 5. Relative velocity with regard to relative distance for $y=0.02 \mathrm{H}$ 


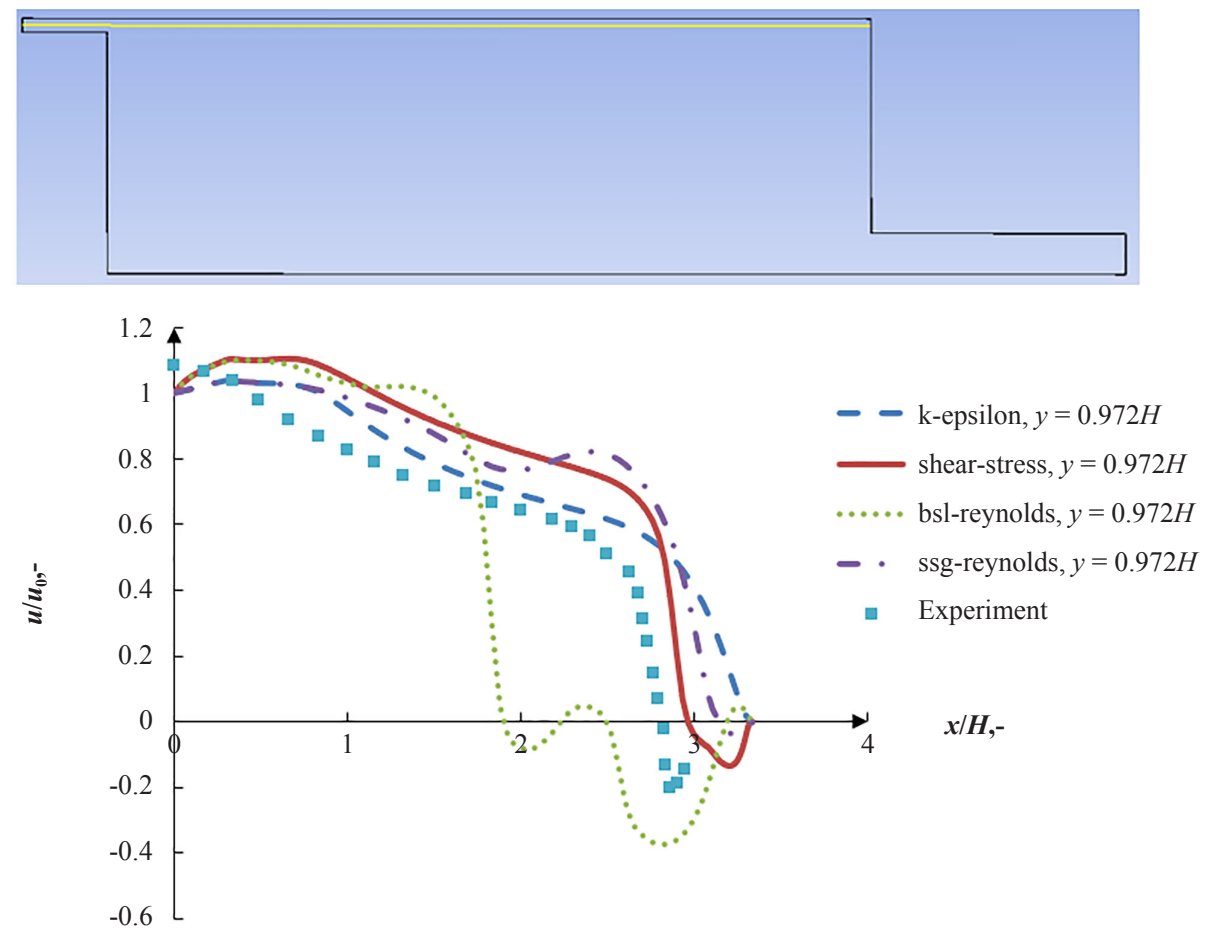

Figure 6. Relative velocity with regard to relative distance for $y=0.972 \mathrm{H}$

Considering the comparison of the velocity profiles obtained for various turbulence models with the experimentally determined profiles, some of the vortices visible in the drawings are non-physical in nature. This is caused by the deficiencies of certain turbulence models used in some of the cases. This applies in particular to vortices visible through stream lines for BSL and SSG models, obtained between two cross-section planes $x=H$ and $x=2 H$.

It seems that the fact of obtaining the worst results, using turbulence models, which are by far the most complicated in calculations, may be associated with the simplification of the model used. This simplification imposed the use of the "symmetry" boundary condition. Combined with a relatively small number of mesh elements, this created the situation of a few mesh elements in the Z-axis direction. Due to the fact that the symmetry condition causes a "mirror image" of the values in combination with the fact of more complicated formulas that more accurately take into account the transfer of stress in different directions, it seems that the use of symmetry could cause error propagation in subsequent iterations. The mutual influence of the size of the numerical mesh, the use of the symmetry condition and the use of more complex turbulence models indicates importance of meshing process on the quality of obtained results.

\section{Error analysis}

Based on Figures 3, 4, 5 and 6, an accuracy of the particular turbulence model has been assessed with an error analysis-Table 1. Average values of absolute errors are presented. The absolute error is defined as:

$$
\Delta e=\left|x_{\text {exp }}-x_{\text {num }}\right|
$$

where $x_{\text {exp }}$ and $x_{\text {num }}$ are the experimental and numerical values.

The error was calculated for 25 measurement points and the corresponding numerical results for each of the turbulence model. The average value of the error was subsequently obtained. 
Table 1. Average errors between the experimental and numerical results

\begin{tabular}{ccccc}
\hline & $\Delta e$ for SSG & $\Delta e$ for BSL & $\Delta e$ for SST & $\Delta e$ for $k-\varepsilon$ \\
\hline$x=H$ & 0.149 & 0.361 & 0.108 & 0.076 \\
$x=2 H$ & 0.113 & 0.207 & 0.080 & 0.058 \\
$y=0.02 H$ & 0.193 & 0.259 & 0.166 & 0.071 \\
$y=0.972 H$ & 0.297 & 0.351 & 0.259 & 0.219 \\
\hline
\end{tabular}

Judging by Table 1, one can notice that the average error is the lowest for the $k-\varepsilon$ approach, whereas it is the highest for the BSL Reynolds approach. The highest errors were found to be near the wall region.

\section{Conclusions}

Based on the simulation results of the air flow in a confined rectangular space and comparing them with the experimental results, one can conclude that for the case under consideration with the Reynolds number equal to 5000 , the most accurate turbulence model is the $k-\varepsilon$ model. This way of thinking is dictated not only by the best mapping of experimental results but also by the lowest amount of iterations needed to achieve convergence of calculations at the required level. However, the accuracy and reliability of the $k-\varepsilon$ approach was substantially diminished close to the wall region. On top of that, the k-e model generated the weakest circulating flow, with respect to the other models.

To sum up, numerical modeling lacks strict mathematical rules regarding the selection of a numerical grid, adapting the turbulence model to the modeled problem and possible simplifications such as the use of the symmetry condition. The accuracy of the selection of these elements is visible "a posteriori" after the simulation. From this, one can draw the general conclusion that the experience of the person performing the simulation is crucial to obtain the results with the required accuracy, while minimizing the time needed to obtain such results.

\section{Conflict of interest}

The authors declare that there is no conflict of interest regarding the publication of this paper.

\section{References}

[1] Djunaedy, E., Loomans, M. G. L. C., and Hensen, J., "Towards a strategy for airflow simulations in building design", in proceedings of the 8th Internatonal conference on Air Distribution in Rooms-Roomvent 2002, 2002, pp. 4-7.

[2] Beausoleil-Morrison, I., "The adaptive coupling of computational fluid dynamics with whole-building thermal simulation", Energy and Buildings, vol. 34, no. 9, pp. 857-871, 2002.

[3] Hensen, J. L. M., Hamelinck, M. J. H., and Loomans, M. G. L. C., "Modelling approaches for displacement ventilation in offices", in 5th International Conference on Air Distribution in Rooms, 1996, pp. 17-19.

[4] Kenneth, S., "Model Answer", Building Services Journal, 2001.

[5] Moser, A., Schalin, A., Off, F., and Yuan, X., "Numerical modelling of heat transfer by radiation and convection in an atrium with thermal inertia," ASHRAE Transactions, vol. 101, no. 2, pp. 1136-1143, 1995.

[6] Negrao, C. O. R., "Conflation of computational fluid dynamics and building thermal simulation", PhD Thesis, University of Strathclyde, Glasgow, U.K, September 1995. Available: http://www.esru.strath.ac.uk/Documents/PhD/ negrao_thesis.pdf.

[7] Zhai, Z., Chen, Q., Klems, J. H. and Haves, P., "Strategies for coupling energy simulations and computational fluid dynamics programs, Building Simulation 2001", in proceedings of the 7th International IBPSA Conference, 2001, pp. $13-15$. 
[8] G. Gunadi, B. Budiarso, A. Siswantara, and A. Daryus, "Turbulence model and validation of air flow in wind tunnel”, International Journal of Technology, vol. 7, no. 8, pp. 1362, 2016.

[9] S. Li, P. Karava, "Evaluation of turbulence models for airflow and heat transfer prediction in BIPV/T systems optimization," Energy Procedia, vol. 30, pp. 1025-1034, 2012.

[10] R. Nagaosa, "Turbulence model-free approach for predictions of air flow dynamics and heat transfer in a fin-andtube exchanger", Energy Conversion and Management, vol. 142, pp. 414-425, 2017.

[11] C. Teodosiu, V. Ilie, R., "Teodosiu, appropriate CFD turbulence model for improving indoor air quality of ventilated spaces", Mathematical Modelling in Civil Engineering, vol. 10, no. 4, 2014.

[12] Bardina, J. E., Huang, P. G., and Coakley, T. J., "Turbulence modeling validation, testing, and development", NASA, Washington D. C., United States, Tech Report, NASA-TM-110446, 1 Apr. 1997.

[13] Menter, F. R., "Two-equation eddy-viscosity turbulence models for engineering applications", AIAA Journal, vol. 32, no. 8, pp. 1598-1605, 1994.

[14] ANSYS CFX-Solver Theory Guide. ANSYS Inc.: Canonsburg, PA, USA, 2009.

[15] Speziale, C. G., Sarkar, S., and Gatski, T. B., "Modelling the pressure-strain correlation of turbulence: An invariant dynamical systems approach", Journal of Fluid Mechanics, vol. 227, pp. 245-272, 1991.

[16] Restivo, A., "Turbulent flow in ventilated rooms", Ph.D. thesis, University of London, March, 1979. 